\title{
Lumbar Spondylolisthesis Progression: What is the Effect of Lumbar Medial Branch Nerve Radiofrequency Ablation on Lumbar Spondylolisthesis Progression? A Single-Center, Observational Study
}

\author{
Trusharth Patel (D) \\ Christopher Watterson ${ }^{2}$ \\ Anne Marie McKenzie- \\ Brown (1D ${ }^{3}$ \\ Boris Spektor ${ }^{3}$ \\ Katherine Egan ${ }^{3}$ \\ David Boorman ${ }^{3}$
}

'Department of Anesthesiology, Division of Pain Management, Emory University School of Medicine, Atlanta, GA, 30308, USA; ${ }^{2}$ Department of Radiology, Emory University School of Medicine, Atlanta, GA, 30308, USA; ${ }^{3}$ Department of Anesthesiology, Emory University School of Medicine, Atlanta, GA, 30308, USA
Correspondence: Trusharth Patel Department of Anesthesiology, Division of Pain Management, Emory University School of Medicine, 550 Peachtree Street NE, Atlanta, GA, 30308, USA

Email trusharth.patel@emory.edu
Background: Radiofrequency ablation (RFA) is a denervation therapy commonly performed for pain of facet etiology. Degenerative spondylolisthesis, a malalignment of the spinal vertebrae, may be a co-existing condition contributing to pain; yet the effect of RFA on advancing listhesis is unknown. To the extent that denervating RFA may weaken paraspinal muscles that provide stability to the spine, the therapy can potentially contribute to progressive spinal instability.

Methods: Single-center, prospective, observational pilot study in an interventional pain practice to test the hypothesis that RFA of painful facets in the setting of spondylolisthesis may contribute to advancement of further degenerative spondylolisthesis. Fifteen participants with pre-existing degenerative Grade I or Grade II spondylolisthesis and coexisting axial lumbar pain underwent lumbar RFA encompassing spondylolisthesis level and followed with post-RFA imaging at 12 months and beyond to measure percent change in spondylolisthesis. Results: The primary outcome was the percent advancement of spondylolisthesis per year measured on post-RFA lateral lumbar spine imaging compared with non-intervention inferred baseline advancement of $2 \%$ per very limited observational studies. Among the 15 participants enrolled, 14 completed the study (median age 66; 64.3\% women; median BMI 33.5; mean follow-up time 23.9 months). The mean advancement of spondylolisthesis per year after RFA was $1.30 \%$ (95\% CI -0.14 to $2.78 \%$ ), with $9 / 14$ below $1.25 \%$.

Conclusion: Among patients with lumbar pain originating from facets in the setting of degenerative spondylolisthesis who underwent lumbar RFA, the observed advancement of spondylolisthesis is clinically similar to the estimated maximum baseline of $2 \%$ per year change. The study findings did not find a destabilizing effect of lumbar RFA in advancing spondylolisthesis in this patient population.

Keywords: degenerative spondylolisthesis, listhesis, radiofrequency ablation, RFA, rhizotomy, denervation, paraspinal muscle, facet, spinal instability

\section{Background}

Spondylolisthesis is a spine condition which refers to a malalignment of the vertebrae of the spine between two adjacent levels. There are numerous causes of spondylolisthesis; its presence can ultimately lead to pain, spinal stenosis, 
neuroforaminal stenosis and instability of the spine by loss of a stabilizing mechanism of the articular processes of the vertebrae. Lower back pain can be a clinical presenting sign; however, the etiology and pathomechanics remain unclear. ${ }^{1}$ Degenerative spondylolisthesis is the most common type of spondylolisthesis; the prevalence varies based on the population studied, ranging anywhere from $7.5 \%$ up to $60 \%$ across different populations. ${ }^{2-5}$ Degenerative spondylolisthesis is reported to be uncommon prior to age 40 for men and 50 for women. ${ }^{6}$ Several risk factors to developing spondylolisthesis have been described: body mass index (BMI), age, and angle of lordosis in women were significantly associated with degenerative spondylolisthesis; increased age was associated in men. ${ }^{5,7}$ Position of imaging, recumbent vs sitting or standing has been proposed as a theoretical association in observing spondylolisthesis; however, neither a large study on an elderly Chinese population nor a 45 -year prospective study found this association. ${ }^{8,9}$

The rate of progression of degenerative lumbar spondylolisthesis remains unclear, as are independent risk factors for progression. Progression of slip in children with pars defects seems to be more prominent in early decades and slows significantly in adulthood at the fourth decade of follow-up evaluation. ${ }^{9}$ One study examined the rate of progression of slippage of vertebrae in 311 patients younger than 30 years old with spondylolisthesis resulting from spondylolysis. Peak slippage in this younger population occurred between the ages of 20-25 years and average slippage per year was calculated to be $0.6 \%$ per year. The authors concluded that slippage was a rare finding overall in this population. ${ }^{10}$ In a small study of 40 Japanese patients aged 34-79 years with degenerative spondylolisthesis, progression greater than $5 \%$ slippage was observed in $30 \%$ with average radiographic observation over 9 years and 2 months. Mean \% slippage or rate in change over time was not reported. The remaining $70 \%$ did not show progression with average observation over 7 years and 10 months. ${ }^{11}$ Matsunaga performed a study on 145 patients with degenerative spondylolisthesis managed non-operatively and found progression in $34 \%$ of patients. Average slip progression over a period of 10-18 years (average 15.8 years) was found to be $15.6 \%$; progression per year was not calculated in this study. ${ }^{12}$ In a group of 190 older men with average age of 74 years and average radiographic follow up of 4.6 years, prevalence of degenerative spondylolisthesis was $30 \%$ and out of this, only $12 \%$ had progression of spondylolisthesis. This study combined their data with the Matsunaga study to give an estimate of spondylolisthesis progression to be approximately $2 \%$ per year $(95 \%$ CI $0.9-4.6 \%)$ in those with baseline spondylolisthesis who developed progression, though this study defined slippage as being greater than $5 \%$ and did not account for those that did not reach 5\%. ${ }^{13}$ Majority of patients did not show progression over the study period, making accurate baseline measures difficult but it can be inferred to be low. Slip progression seems to be most prevalent between the L4-L5 level according to a 15-year study; risk for slip progression was greatest for those between 40-60 years of age compared with those older than $60 .{ }^{14}$

\section{Objective}

We present a study examining the effect of lumbar medial branch RFA on patients with chronic lumbar pain originating from facets in the setting of Grade I or II lumbar spondylolisthesis. Facet-mediated pain may be present with degenerative spondylolisthesis through a presumed ventral-dorsal shearing stress on facets. RFA that denervates lumbar facets may offer pain relief, but the spinal stabilizing effect of this therapy in such a population remains unknown. Medial branch nerves are targeted for thermal neurolysis in this therapy; however, ablation of adjacent branching nerves from the common dorsal ramus cannot always be avoided. These nerves are the intermediate and lateral branch nerves which provide innervation to the paraspinal muscles, longissimus and iliocostalis. ${ }^{15}$ The other major lumbar paraspinal muscles, multifidus, are innervated by the medial branch nerves after splitting off from branches innervating the facet joint. ${ }^{16}$ Transient muscle atrophy of the multifidus muscles after successful medial branch RFA has been demonstrated on MRI studies, though it is unclear why bilateral atrophy was seen on imaging when only unilateral RFA was performed. There was also lack of agreement among the blinded radiologists on levels treated, making any correlation difficult. These muscles, with predominantly slow twitch fibers, serve as postural stabilizers of the spine. ${ }^{17}$ Standard thermal RFA lesioning at $80^{\circ} \mathrm{C}$ for $90 \mathrm{~s}$ with a standard 20- to 18-gauge monopolar radiofrequency needle has been shown to have a transverse distance of 5.3-5.9 mm, respectively. This may encompass branch nerves innervating paraspinal muscles, more so those innervating the multifidus muscle; however, no long-term functional adverse effect has been demonstrated. ${ }^{17,18}$ It was our hypothesis that lumbar medial branch nerve 
RFA in patients with pre-existing degenerative spondylolisthesis would not result in advancement of listhesis postRFA observed over at least a 12-month period.

\section{Methods}

In the course of usual treatment in an outpatient chronic pain clinic for lower back pain of facet etiology after exhausting conservative measures, 15 patients over age 40 with ongoing back pain and observed Grade I or Grade II spondylolisthesis who underwent or were planned to undergo lumbar medial branch nerve radiofrequency ablation from 2014-2018 were prospectively selected for observational study participation. Approval was obtained from the Institutional Review Board for Medical Ethics to recruit and treat patients who met the selection criteria. All participants had baseline imaging within 4 months of RFA in which there were clear lateral lumbosacral views for accurate measurement of baseline spondylolisthesis for comparison. Post-procedure imaging with lateral views through the lumbosacral spine was obtained at greater than 12 months. Of the 15 patients, one patient was not able to complete post-procedure imaging. A total of 14 patients completed the study.

Pre-procedure imaging included either those from the pre-RFA fluoroscopic diagnostic nerve block, fluoroscopic images from the RFA procedure, CT, MRI, or plain lumbar $\mathrm{X}$-ray at baseline. Fluoroscopic images used were devoid of rotational artifact. Post-RFA lumbar X-rays with lateral views through the lumbosacral junction were obtained on all patients and funded from the study grant. However, if a CT or MRI was obtained for non-study purposes and exceeded 12 months post-ablation, this was used for spondylolisthesis measurement. This was done to minimize variability in spondylolisthesis due to weight-bearing changes between recumbent and standing position. All images were in neutral position and no dynamic imaging was performed. When fluoroscopic images were utilized for measurement, measurements were calibrated by comparing same-level vertebral body height to vertebral height on available radiographs, CTs, or MRIs. This was done to control for variable degrees of magnification inherent to the fluoroscopic modality. All participants were provided remuneration for the follow-up X-rays. Medial branch nerve radiofrequency ablation was performed by experienced interventional pain specialists using a parallel needle placement technique. ${ }^{15}$ Standard Stryker equipment including 18-gauge needles $100-150 \mathrm{~mm}$ in length with a standard 10-mm active tip was used and a standard thermal lesioning temperature of $80^{\circ} \mathrm{C}$ for $90 \mathrm{~s}$ was performed. All participants had symmetrical, bilateral RFA performed. All patients only had one RFA procedure performed during the study period but may have had previous ablation procedures prior to the study.

A single, experienced neuromuscular radiologist analyzed all pre- and post-RFA images and measured listhesis in millimeters based on the displacement of the caudal endplate of the superior vertebral level relative to the adjacent inferior vertebral level. A departmental biostatistician provided the statistical analysis.

\section{Statistical Methodology}

A statistical power sensitivity analysis was conducted using G*Power 3.1.9.4. ${ }^{19}$ Performing a one-tailed, onesample $t$-test with $\mathrm{n}=14$, alpha $=0.05$ and power $=0.80$, a sample size of 14 is able to detect a "medium to large" effect size of 0.70 ("Medium" $=0.5$, "Large" $=0.8$ ).

Statistical analysis was performed by SAS 9.4 (Cary, NC). Data from 14 patients were obtained. The main outcome, percent advancement in listhesis per year, was calculated as:

[(Post RFA - Pre RFA)/Endplate Distance] * 100\% * (12 Months/Months Follow-up)

A positive number indicates listhesis advancement; a negative number indicates an improvement or measurement variability.

Comparisons of demographics and potential confounders against the outcome were performed with nonparametric tests, to avoid assumptions of normal distribution: Spearman rank Correlation (instead of Pearson Correlation), Mann-Whitney $U$-test (instead of the $t$-test), and Kruskal-Wallis test (instead of ANOVA). Because this study's main hypothesis is that there is no adverse effect of RFA on listhesis, we are effectively trying to prove the null hypothesis, and a traditional hypothesis test cannot be performed. Instead, we are performing a Method Comparison study, with the outcome descriptive statistics of the $95 \%$ confidence interval, compared against an inferred "natural" decline of a maximum $2 \%$ as described in a study by Denard combining their data with the Matsunaga study data. It should be noted that actual calculations to produce a baseline progression of $2 \%$ were not detailed in the Denard study and an assumption is made that it is at a maximum of $2 \%$, factoring in that the majority of patients during the observation period did not have progression and those with less than $5 \%$ progression were excluded in calculations. ${ }^{12,13}$ 
Table I Demographics and Outcome Measure in \% Advancement in Listhesis

\begin{tabular}{|c|c|c|c|c|}
\hline Continuous Variables & \multicolumn{2}{|c|}{ Median (IQR) } & Mean (SD) & p-value \\
\hline BMI & \multicolumn{2}{|c|}{$33.50(29.5-36.4)$} & & $0.53^{\mathrm{a}}$ \\
\hline Age & \multicolumn{2}{|l|}{$66(55-70)$} & & $0.099^{a}$ \\
\hline Pre-RFA Listhesis (mm) & \multicolumn{2}{|l|}{$4.65(3.7-5.8)$} & & $0.90^{\mathrm{a}}$ \\
\hline Post-RFA Listhesis (mm) & \multicolumn{2}{|l|}{$5.00(4.2-6.3)$} & & $\begin{array}{l}P=0.0086^{\circ} \\
r=0.67\end{array}$ \\
\hline Follow-up Time (months) & \multicolumn{2}{|l|}{$23.50(17-27)$} & $23.86(9.03)$ & $0.15^{a}$ \\
\hline Percent advancement/year & \multicolumn{2}{|c|}{$0.72(-0.50$ to 3.07$)$} & $1.30(2.55)$ & $0.12^{b}$ \\
\hline Categorical Variables & $\mathbf{I}$ & 2 & 3 & p-value \\
\hline Gender & $\begin{array}{l}\text { Female } \\
9(64.3 \%)\end{array}$ & $\begin{array}{l}\text { Male } \\
5(35.7 \%)\end{array}$ & & $0.60^{c}$ \\
\hline Race & $\begin{array}{l}\text { African Am. } \\
10(76.9 \%)\end{array}$ & $\begin{array}{l}\text { Caucasian } \\
3(23.1 \%)\end{array}$ & $\begin{array}{l}\text { Unknown } \\
\text { I (NA) }\end{array}$ & $0.68^{c}$ \\
\hline Prior RFA & $\begin{array}{l}\text { No } \\
9(64.3 \%)\end{array}$ & $\begin{array}{l}\text { Yes } \\
5(35.7 \%)\end{array}$ & & $0.90^{\mathrm{c}}$ \\
\hline Vertebrae Listhesis & $\begin{array}{l}\text { L4-L5 } \\
9(64.3 \%)\end{array}$ & $\begin{array}{l}\text { L5-SI } \\
5(35.7 \%)\end{array}$ & & $0.44^{\mathrm{c}}$ \\
\hline Imaging & $\begin{array}{l}\text { L-spine MRI } \\
3(21.4 \%)\end{array}$ & $\begin{array}{l}\text { L-spine X-ray } \\
4(28.6 \%)\end{array}$ & $\begin{array}{l}\text { Proc. Fluoro } \\
7(50 \%)\end{array}$ & $0.33^{\mathrm{d}}$ \\
\hline Mismatched imaging pre- and post-RFA & $\begin{array}{l}\text { No } \\
10 \text { (7I.4\%) }\end{array}$ & $\begin{array}{l}\text { Yes } \\
4(28.6 \%)\end{array}$ & & $0.10^{c}$ \\
\hline
\end{tabular}

Notes: ${ }^{a}$ Spearman Rank Correlation, equivalent to linear regression. ${ }^{b}$ Signed Rank test, equivalent to the one-sample $t$-test. ${ }^{c}$ Mann-Whitney $U$-test, equivalent to the twosample t-test. ${ }^{d}$ Kruskal-Wallis test, equivalent to ANOVA.

Abbreviations: IQR, interquartile range; SD, standard deviation; RFA, radiofrequency ablation.

\section{Results}

Table 1 shows medians and interquartile range or frequencies for several demographic variables (age, gender, race, BMI). Using non-parametric statistical tests, there was no significant difference in percent advancement in listhesis per year by demographics: age, gender, race or BMI. There was also no significant difference by pre-RFA listhesis score, prior RFA, vertebrae involved, imaging technique, or mismatch in recumbency for comparative images. Additionally, using Spearman Rank Correlation, age was significantly negatively correlated with BMI $(\mathrm{p}=0.0029, \mathrm{r}=-0.73)$ and follow-up time $(\mathrm{p}=$ $0.029, \mathrm{r}=-0.58)$. Post-RFA listhesis was positively correlated with pre-RFA listhesis $(p=0.013, r=0.64)$ and percent advancement of listhesis per year $(\mathrm{p}=0.0086, \mathrm{r}=0.67)$.

For the primary outcome, percent advancement of listhesis per year, $9(64 \%)$ had values less than the comparison $2 \%$ per year without intervention, with the highest value of this subset $1.25 \%$. Figure 1 shows the outcome by pre-RFA levels. Data for the outcome were slightly positively skewed, but multiple tests for normality showed it could be treated as normally distributed, including the Shapiro-Wilks test ( $\mathrm{p}=$ 0.69). This yields a mean percent advancement of listhesis per year of $1.30 \%$ (95\% CI -0.17 to 2.78$)$, assuming normality.

\section{Primary Outcome}

The goal was to determine if there was advancement in listhesis measured in percent change per year over the comparison $2 \%$ per year baseline described in the Denard study. Data for the percent change per year was normally distributed. No significant change was found $(\mathrm{p}=$ $0.79)$. The mean change was $1.30 \%(95 \% \mathrm{CI}-0.14$ to $2.78 \%)$. Out of 14 patients, $8(57 \%)$ had any advancement of listhesis over time (max: 70\%), and 5 of the 8 had advancement greater than $2 \%$ comparison baseline estimate. These patients had a median change of $3.48 \%$, a minimum of $2.99 \%$ and a maximum of $6.50 \%$. Out of 14 patients, $6(43 \%)$ had slight reduction in listhesis (min: 


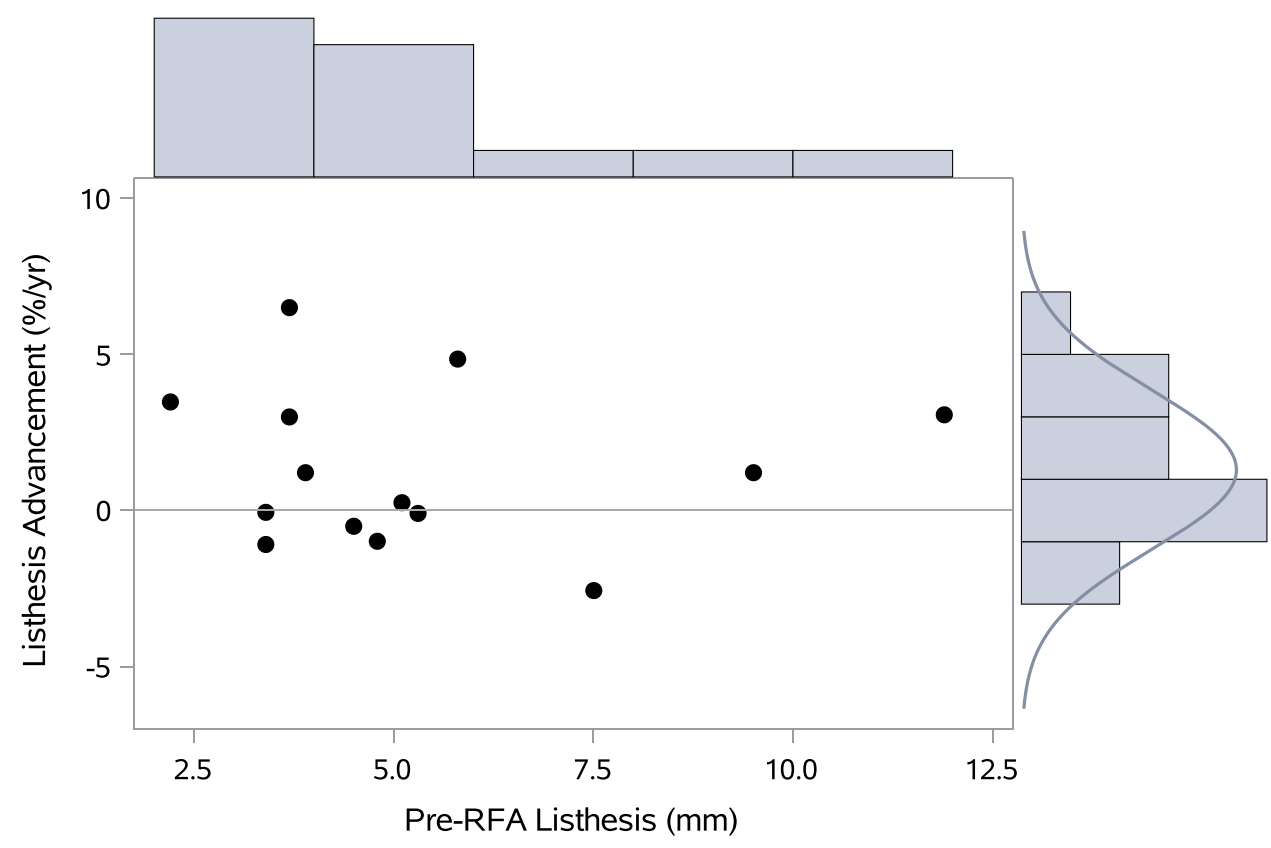

Figure I Scatterplot of the percent advancement in listhesis score per year as a function of the pre-RFA score. Positive numbers indicate an advancement of listhesis. Six patients (43\%) saw a slight improvement in listhesis while an additional 3 patients $(21 \%)$ showed advancement less than I.25\%, i.e. below $2 \%$, the expected decline without intervention. Five patients (36\%) saw an advancement of listhesis beyond $2 \%$.

$-12 \%)$, with one patient having mismatch in recumbency on imaging. This study shows that performing RFA advances listhesis a mean of $1.30 \%$ per year with an upper limit of confidence interval of $2.78 \%$. Of the 14 patients, 4 patients that had a mismatch in recumbency position between pre-RFA and post-RFA imaging collectively did not demonstrate a change in listhesis. Of the 14 patients, 2 patients had both pre- and post-RFA lumbar MRI imaging used for listhesis measurements. In both cases, listhesis was actually slightly improved post-RFA but this could easily be attributed to the margin of error with measurement. Both cases also showed some baseline fatty infiltration in the multifidus muscles in crosssectional images across the L4/5 and L5/S1 intervertebral space levels; significant increase in fatty infiltrates postRFA to suggest muscle atrophy was not observed.

\section{Discussion}

The overall mean percent change of listhesis per year we observed was $1.30 \%$ (95\% confidence interval -0.17 to 2.78). This included patients who saw no change or a slight improvement in listhesis. One large observational study estimated the percent advancement of degenerative spondylolisthesis to be $2 \%$ per year in a small percentage of patients with baseline spondylolisthesis. To further obfuscate the matter, those with less than 5\% slippage were excluded in calculations. $^{13}$ The actual baseline progression from observational studies is likely lower if taking into consideration those with less than $5 \%$ progression were not included in calculations and the majority of patients with spondylolisthesis observed over many years did not show progression. ${ }^{12,13}$ We saw a progression of listhesis in 5 patients out of 14 (35.7\%) with baseline spondylolisthesis, with an assumption of a meaningful advancement occurring beyond $1.25 \%$ per year. Nine patients did not meet this level of progression, despite having had the RFA intervention. Looking at just the 5 patients with meaningful progression, we calculated a median progression of $3.48 \%$ per year (range: $2.99-6.50 \%$ ) in $35.7 \%$ of patients. Matsunaga observed a slip progression rate of $15.6 \%$ over 15.8 years in $34 \%$ of non-operative patients with degenerative spondylolisthesis. ${ }^{12}$ We conclude that our overall findings are similar to the natural slip progression of degenerative spondylolisthesis of a maximum of $2 \%$ as best as we could identify among limited medical evidence. However, a small group of patients who do show progression, may develop greater advancement of listhesis having had RFA, though more robust data are needed to conclude this. The actual baseline progression rate without intervention remains unclear and likely is not a linear progression. The neuroablative procedure, lumbar medial branch nerve RFA does not appear to destabilize the spine by advancing spondylolisthesis in this patient population 
based on observed mean advancement of spondylolisthesis being largely similar to non-surgical patients. Some paraspinal muscle atrophy may occur post-procedure as shown in a small study of 5 patients with some inconsistencies in findings. ${ }^{17}$ Two patients with MRI imaging in our study had minimal baseline fatty infiltrates of the multifidus muscles but this did not significantly change post-RFA (21-34 months). Advancement of spondylolisthesis was not seen in these 2 patients. We cannot refute nor support this as a mechanism of advancing listhesis with the small sample size for analysis.

We did observe a slight decrease in spondylolisthesis over time in 6/14 (43\%) of patients. We believe that this may due to several factors: small margin of error in measurement of listhesis that is in the order of millimeters; variability in imaging resolution; calibration across the different imaging modalities used. Improvement in spondylolisthesis may theoretically occur with strengthening of lumbar paraspinal and abdominal muscles as studies associate weakening or atrophy of these muscles to developing degenerative spondylolisthesis..$^{20,21}$

Most patients in our study had greatest listhesis over L4-5 level which is consistent with previous epidemiological studies. ${ }^{27,22}$ Median BMI in our study was 33.5 which is categorized as obese. BMI as a risk factor for degenerative spondylolisthesis remains controversial. ${ }^{5,7}$ It has been theorized that excessive weight may exacerbate load and shearing forces on the spine and contribute to degenerative spondylolisthesis. Conversely, BMI may also contribute to degenerative disc disease, facet overlap with osteophytosis, and ossification of ligaments which may facilitate stabilization of spondylolisthesis. ${ }^{1,23}$ The ratio of female:male in our study was 9:5. Though our sample size was too small to confirm the prevalence gender ratios reported in other studies, it is in accord with epidemiological studies reporting degenerative spondylolisthesis having a greater prevalence in women., ${ }^{4,7}$

\section{Study Limitations}

This study has several limitations. First, the sample size is small. Hundreds of patients were screened through our center to identify patients with lumbar pain originating from facets undergoing RFA with coexisting spondylolisthesis and good quality baseline imaging within 4 months of RFA. Several international studies report a low prevalence of degenerative spondylolisthesis ranging from $12 \%$ up to $30 \%$. This may be observed in the general
American population as well and a small percentage will have pain, specifically of facet etiology. Second, our study is from a single institution. Current findings thus reflect on the clinical practices of the institution, which may limit the generalizability of the results. Third, in some patients, the pre- and post-RFA imaging was in different recumbency positioning which may affect observed spondylolisthesis to a small degree. This still remains controversial in the medical literature. ${ }^{5,8}$ We did not observe any correlation in spondylolisthesis in the 4 patients with mismatch imaging. Fourth, there remain limited data on the accepted rate of advancement of degenerative spondylolisthesis per year as a baseline comparison. We found a single study which reported such data. Many patients with a small degree of listhesis $(<5)$ were excluded and method of calculations combining their study with another were not clearly reported. ${ }^{13}$ These factors can obfuscate comparison studies such as ours. Also, we are implicitly assuming a continuous linear progression of listhesis over time. In reality, the rate of degenerative spondylolisthesis progression may be quite variable with numerous contributing factors. Periods of peak progression have been described in prospective observational pediatric populations as well as older adult studies. ${ }^{9,14}$ Conversely, a slowing of progression or stabilization of spondylolisthesis has been described in the very elderly population. This was also observed in our study in which $9 / 14$ with baseline spondylolisthesis did not show advancement of slippage beyond $1.25 \%$. However, our median follow up period was 23.5 months, arguably not long enough to capture those with a very slow rate of progression of listhesis, assuming a somewhat continuous progression over time.

\section{Conclusion}

Among patients with degenerative lumbar spondylosis with coexisting facetogenic pain who underwent medial branch nerve radiofrequency ablation, a mean spondylolisthesis advancement of $1.3 \%$ per year is comparable to the estimated maximum rate of natural progression without any intervention. Radiofrequency ablation in this patient population may be generally considered a safe therapy as a strategy for pain management. However, a small percentage of patients who do experience progression of spondylolisthesis may progress to a greater degree after having had RFA. Larger studies with longer followup periods are needed to confirm the study's findings. 


\section{Abbreviations}

RFA, radiofrequency ablation; BMI, body mass index; $\mathrm{IQR}$, interquartile range; SD, standard deviation.

\section{Data Sharing Statement}

The datasets during and/or analyzed during the current study are available from the corresponding author on reasonable request.

\section{Ethics Approval and Consent to Participate}

The authors declare that the work described has been carried out in accordance with the Declaration of Helsinki of the World Medical Association revised in 2013 for experiments involving humans. Approval to conduct this study was obtained from Emory University School of Medicine IRB (IRB ref \#: 00082388). The authors declare that this report does not contain any personal information that could lead to the identification of the patient(s).

\section{Ethics and Consent to Participate}

The authors declare that they obtained a written informed consent from the patients and/or volunteers included in the article. The authors also confirm that the personal details of the patients and/or volunteers have been removed.

\section{Author Contributions}

All authors made substantial contributions to conception and design, acquisition of data, or analysis and interpretation of data; took part in drafting the article or revising it critically for important intellectual content; agreed to submit to the current journal; gave final approval of the version to be published; and agree to be accountable for all aspects of the work.

\section{Funding}

Funding for this study was from internal departmental funding award. Funding source played no role in the design or conduct of this study.

\section{Disclosure}

The authors declare that they have no competing interests.

\section{References}

1. Kalichman L, Hunter DJ. Degenerative lumbar spondylolisthesis: anatomy, biomechanics and risk factors. J Back Musculoskelet Rehabil. 2008;21(1):1-12. doi:10.3233/BMR-2008-21101
2. He LC, Wang YXJ, Gong JS, et al. Prevalence and Risk factors of lumbar spondylolisthesis in elderly Chinese men and women. Eur Radiol. 2014;24(2):441-448. doi:10.1007/s00330-013-3041-5

3. Sanderson PL, Fraser RD. The influence of pregnancy on the development of degenerative spondylolisthesis. $J$ Bone Joint Surg Br. 1996;78-B(6):951-954. doi:10.1302/0301-620X.78B6.0780951

4. Vogt MT, Rubin DA, Valentin RS, et al. Lumbar olisthesis and lower back symptoms in elderly white women. The Study of Osteoporotic Fractures. Spine. 1998;23(23):2640-2647.

5. DeVine JG, Schenk-Kisser JM, Skelly AC. Risk factors for degenerative spondylolisthesis: a systematic review. Evid Based Spine Care J. 2012;3(2):25-34. doi:10.1055/s-0031-1298615

6. Kalichman L, Kim DH, Li L, Guermazi A, Berkin V, Hunter DJ. Spondylolysis and spondylolisthesis: prevalence and association with low back pain in the adult community-based population. Spine. 2009;34(2):199-205. doi:10.1097/BRS.0b013e31818edcfd

7. Jacobsen S, Sonne-Holm S, Rovsing H, Monrad H, Gebuhr P. Degenerative lumbar spondylolisthesis: an epidemiological perspective the copenhagen osteoarthritis Study. Spine. 2003;32(1):120-125. doi:10.1097/01.brs.0000250979.12398.96

8. Yxj W, Deng M, Leung CS, Ahuja AT, Kwok T, Leung PC. Lumbar spondylolisthesis progression and de novo spondylolisthesis in elderly Chinese men and women. Spine. 2016;41(13):1096-1103. doi:10.1097/BRS.0000000000001507

9. Beutler WJ, Fredrickson BE, Murtland A, Sweeney CA, Grant WD, Daniel Baker D. The natural history of spondylolysis and spondylolisthesis 45-year follow-up evaluation. Spine. 2003;28 (10):1027-1035. doi:10.1097/01.BRS.0000061992.98108.A0

10. Danielson BI, Frennered AK, Irstam LKH. Radiographic progression of isthmic lumbar spondylolisthesis in young patients. Spine. 1991;16 (4):422-425. doi:10.1097/00007632-199104000-00005

11. Matsunaga S, Sakou T, Morizono Y, Masuda A, Demirtas M. Natural history of degenerative spondylolisthesis pathogenesis and natural course of slippage. Spine. 1990;15(11):1204-1210. doi:10.1097/ 00007632-199011010-00021

12. Matsunaga S, Ijiri K, Hayashi K. Nonsurgically managed patients with degenerative spondylolisthesis: a 10- to 18-year follow-up study. J Neurosurg. 2000;93(2 Suppl):194-198. doi:10.3171/spi.2000.93.2.0194

13. Denard PJ, Holton KF, Miller J, et al. Lumbar spondylolisthesis among elderly men: prevalence, correlates and progression. Spine. 2010;35(10):1072-1078. doi:10.1097/BRS.0b013e3181bd9e19

14. Enyo Y, Yoshimura N, Yamada H, Hashizume H, Yoshida M. Radiographic natural course of lumbar degenerative spondylolisthesis and its risk factors related to the progression and onset in a 15-year community-based cohort study: the Miyama study. J Orthop Sci. 2015;20(6):978-984. doi:10.1007/s00776-015-0759-8

15. Lau P, Mercer S, Govind J, Bogduk N. The surgical anatomy of lumbar medial branch neurotomy (Facet Denervation). Pain Med. 2004;5(3):289-298. doi:10.1111/j.1526-4637.2004.04042.x

16. Cohen SP, Bhaskar A, Bhatia A, et al. Consensus practice guidelines on interventions for lumbar facet joint pain from a multispecialty, international working group. Reg Anesth Pain Med. 2020;45:424-467. doi:10.1136/rapm-2019-101243

17. Dreyfuss P, Stout A, Aprill C, Pollei S, Johnson B, Bogduk N. The significance of multifidus atrophy after successful radiofrequency neurotomy for low back pain. AAPM\&R. 2009;1:719-722. doi:10.1016/j.pmrj.2009.05.014

18. Cedeño DL, Vallejo A, Kelley CA, Tilley DM, Kumar N. Comparisons of lesion volumes and shapes produced by a radiofrequency system with a cooled, a protruding, or a monopolar probe. Pain Physician. 2017;20(20;6):E915-E922. (). doi:10.36076/ppj.20.5.E915

19. Faul F, Erdfelder E, Lang AG, Buchner A. G*Power 3: a flexible statistical power analysis program for the social, behavioral, and biomedical sciences. Behav Res Methods. 2007;39(2):175-191. doi:10.3758/BF03193146 
20. Fraser RD, Brooks F, Dalzell K. Degenerative spondylolisthesis: a prospective cross-sectional cohort study on the role of weakened anterior abdominal musculature on causation. Eur Spine J. 2019;28 (6):1406-1412. doi:10.1007/s00586-018-5758-y

21. Hiyama A, Katoh H, Sakai D, Tanaka M, Sato M, Watanabe M. The correlation analysis between sagittal alignment and cross-sectional area of paraspinal muscle in patients with lumbar spinal stenosis and degenerative spondylolisthesis. BMC Musculoskelet Disord. 2019;20 (1):260-352. doi:10.1186/s12891-019-2733-7
22. Wang YXJ, Kaplar Z, Deng M, Leung JCS. Lumbar degenerative spondylolisthesis epidemiology: a systematic review with a focus on gender-specific and age-specific prevalence. J Orthop Transl. 2017;11:39-52.

23. Kirkaldy-Willis WH, Farfan HF. Instability of the lumbar spine. Clin Ortho. 1982;162:110-123.

\section{Publish your work in this journal}

The Journal of Pain Research is an international, peer reviewed, open access, online journal that welcomes laboratory and clinical findings in the fields of pain research and the prevention and management of pain. Original research, reviews, symposium reports, hypothesis formation and commentaries are all considered for publication. The manuscript

Submit your manuscript here: https://www.dovepress.com/journal-of-pain-research-journal management system is completely online and includes a very quick and fair peer-review system, which is all easy to use. Visit http:// www.dovepress.com/testimonials.php to read real quotes from published authors. 